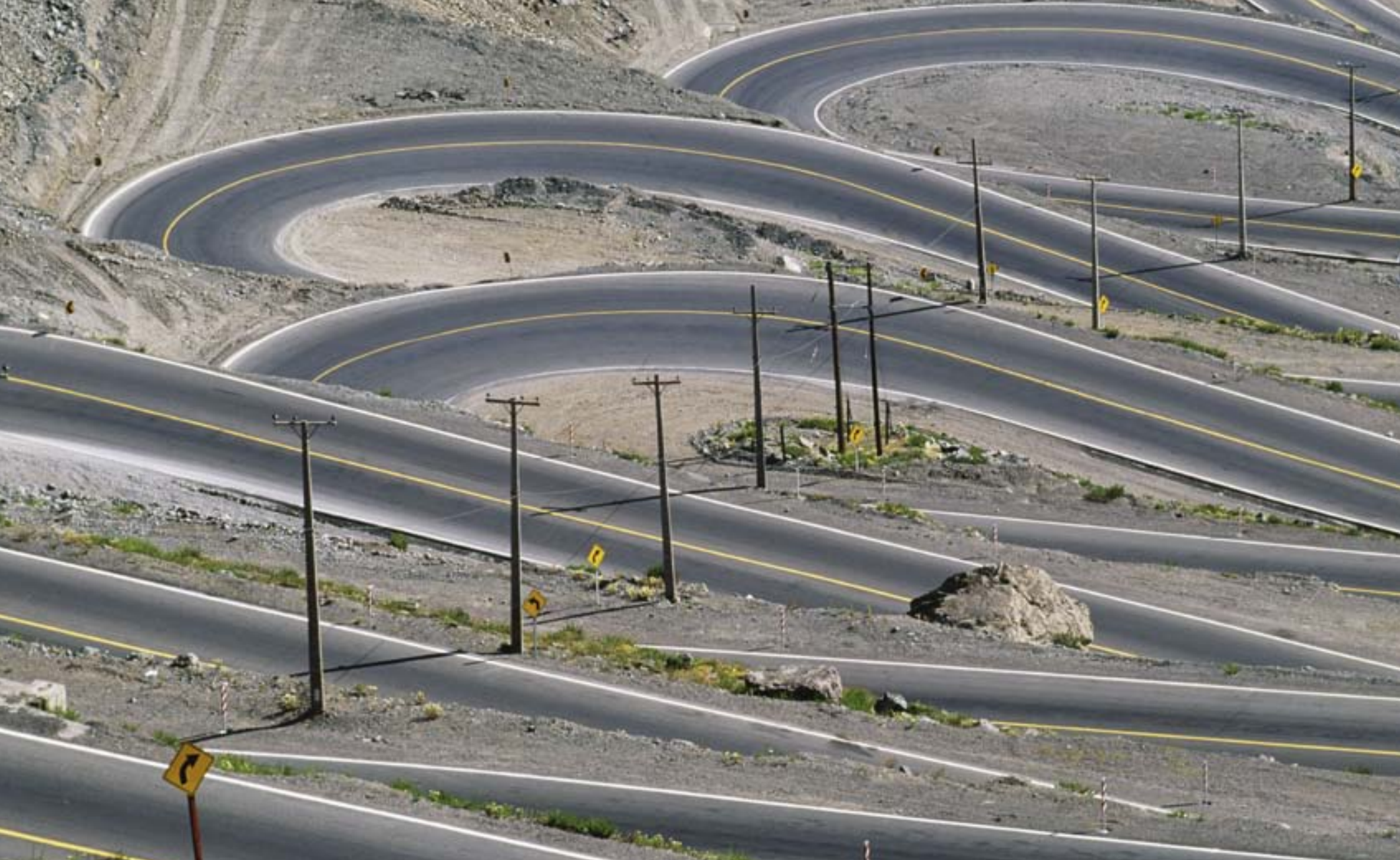

\section{Modernidad coordinada}

$¿$ Van de la mano la modernidad y las inversiones públicas? En lo que se refiere al transporte, solo si están coordinadas. Por Eduardo Saavedra P. *

Doctor en Economía de Cornell University. Académico de la Facultad de Economia y Negocios de la Universidad Alberto Hurtado. Director del Master of Arts ILADESGeorgetown University.

version ejecutada por el Ministerio de Obras Publicas (MOP) desde 1995 en adelante, junrepresentan por sobre el 80\% de las inversiones totales.

Los mayores gastos en infraestructura inversión decido en "vialidad", es decir en por el sector privado a través de litaciones de las obras. Interesante resulta observar
Inversiones del MOP 95-08 (billones de \$de dic. 08)

0,5

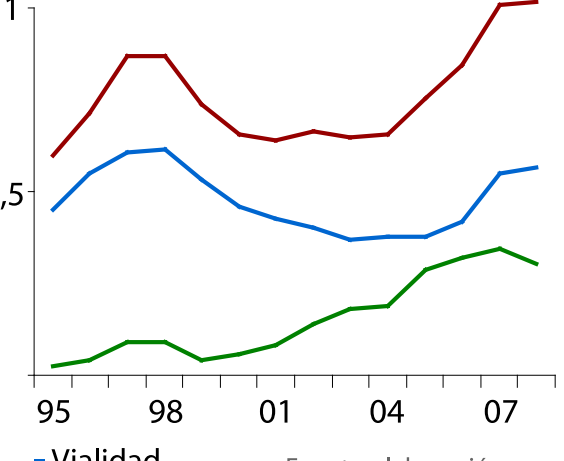

$\begin{array}{ll}\text { R.Vialidad } & \text { Fuente: elaboración } \\ \text { "- Toncesiones } & \text { propia basadación } \\ \text { - Total anual } & \text { en información del MOP }\end{array}$

importancia creciente de las inversiones a través del sistema de "concesiones": I uego de licitada, la obra es construida y operada por los propios privados

Entre 1998 y 2005, mientras aumentaba este medio de participación mixta, se reducia en casi igual proporción la inversión a traves de medio más tradicional. Por lo tantor aún cuando dependan del mismo ministerio, jocutar importantes inversiones en transporte, lo que inevitablemente nos plantea preguntas respecto de cuán coordinadas están las decisiones de qué obras se ejecutará cómo se evalúan y sobre todo, qué unida to hace, cómo se controla la construcción de las obras y, en el caso de las concesiones, su operación privada bajo los estándares establecidos en la licitación.

Estos posibles problemas de coordinación y evaluación de las inversiones bajo estándares comunes, y la fiscalización de su construcción y de la operación posterior se exacerba si tenemos presente que hay emEnap y varios puertos que además realizan sus propias inversiones en infraestructura de transporte.

Las inversiones en infraestructura de transporte de las empresas públicas han estado en torno al $20 \%-25 \%$ de la inversión
en transporte que ha realizado el MOP en entos últimos 15 años. Las inversiones de estos ultimos 15 años. Las inversiones de
estas empresas debieran ser aprobadas en el sistema nacional de inversiones, unidad dependiente del MIDEPLAN, pero nada esta establecido en la ley respecto de cómo de- ben coordinarse con aquellas que realiza . De facto, aun cuando debiera ser tarea del Ministerio de Transportes y Telecomunitravés de la Dirección de Presupuestos, órgano que ha hecho esta tarea de coor nación, án cuando bien sabemos que Hacienda no es el mejor orientador: su rol es financiro no técnico. en a coordinación tienen que ver con inversiones en ferrocarriles versus carreteras erurbanas, en Metro versus vias urbanas, puertos versus infraestructura vial y feroviaria. Si se consideran, además, casos de social no estuvo presente, como los de infraestructura ferroviaria o nuestro "casi" puente sobre el canal de Chacao, la realidad nos hace pensar que algo más se requiere hacer para que la inversion publica vaya de la mano con a modernidad pública.

El pais debe tener una unidad encargada de la coordinación de las inversiones en infraestructura de transporte, que evade los ministerios que proponen realizar esa obras

Si se ha observado, además, carencias en fals de indende las abras, y por sobre todo gada de fiscalizar la operación de las obras concesionadas, entonces nuestra propuesta es dotar precisamente a esta entidad de poder suficiente para acometer todas esas tareas. Se trata, de alguna manera de un suprarregulador del transporte público en los ámbitos propios de la coordinación, evauación, fiscalización y regulación de la infraestructura correspondiente. Bajo que miisterio quede la tutela de este organismo es irrelevante: podria ser perfectamente el MOP IMT; lo que importa es que le otorgue poder de realizar las tareas encomendadas asi evitar inversiones de dudosa rentabilidad implementación ministerio, aunque regerada por el propio el gráfico

CONFLICTIVOS INTERESES

IUF hasta un año
-UF más de un año. Hasta 2000 UF.
-UF más de un año. Sobre 2000 UF. $8,5 \%$

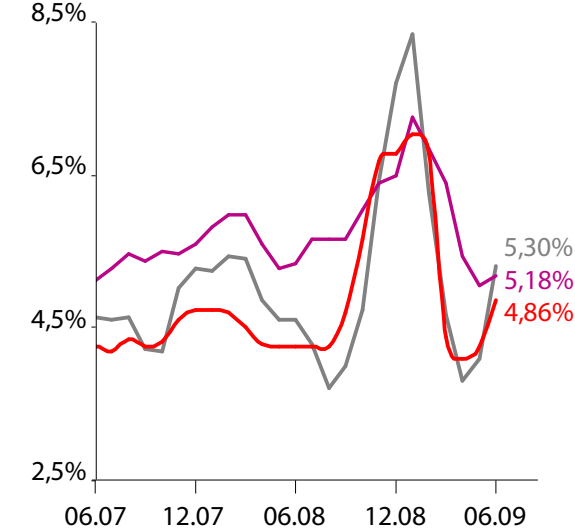

Juan Foxley

El gráfico ilustra las operaciones en moneda nacional reajustable desde el segundo semestre de 2007. Las ta-

sas reales de colocación cobradas en promedio por los bancos durante el primer semestre de 2009 son similares llamada crisis sub-prime.

Si bien es cierto que hubo una leve alza hacia junio, probablemente en reacción a la aparición de tasas de inflación negativas, las tasas en UF son de 2008, independiente de los montos y de los plazos.

A su turno, el spread neto (margen entre tasas de prestamos y captación menos el costo del encaje) había aumentado levemente. Desde 1,83 en agosto de 2008 a 1,99 en julio de 2009, para operaciones en UF entre 90 clas y un ano.

Tos números no creciente en elasceso a crito embargo, es probable aue deudores con mala o desconocida reputación de repago enfrenten las dificultades propias de una economia que ha aumentado su riesoo, mientras que, por supuesto, se mantienen las regulaciones prudenciales que protegen a los depositantes. menores a las observadas en diciembre

rol en la definiculador no jugar proyecto (a lo más sugerir modificacion que reduzcan las fallas de coordinación), y acuerdo a lo requerido. 